\title{
A CONDUTA DIETOTERÁPICA NO TRATAMENTO DA DOENÇA DO REFLUXO GASTROESOFÁGICO - RELATO DE CASO
}

\author{
Tatiane dos Santos Aparecido-Gonçalves ${ }^{1 *}$ \\ Patrícia Franco Goncalves Previato do Amaral ${ }^{2}$ \\ Andréia Assunção Soares ${ }^{3}$ \\ Maressa de Lima Rodrigues ${ }^{4}$ \\ Laura Maria Soares Betineli \\ Ricardo de Melo Germano ${ }^{6}$
}

\begin{abstract}
APARECIDO-GONÇALVES, T. dos S.; AMARAL, P. F. G. P. do; SOARES, A. A.; RODRIGUES, M. de L.; BERTINELI, L. M. S.; GERMANO, R. de M. A conduta dietoterápica no tratamento da doença do refluxo gastroesofágico - relato de caso. Arq. Cienc. Saúde UNIPAR, Umuarama, v. 20, n. 3, p, 199-203, set./dez. 2016.

RESUMO: A doença do refluxo gastroesofágico é causada pela redução da pressão do esfíncter esofágico inferior e pode ser agravado por fracionamento inadequado da dieta e pela produção de espécies reativas ao oxigênio. Os tratamentos medicamentosos e cirúrgicos se destacam, contudo, existem opções de terapêutica não farmacológica, como as vitaminas e minerais com funções antioxidantes, atuam como adjuvantes neste tipo de tratamento. Portanto, este trabalho teve como objetivo avaliar as funções das vitaminas e minerais suplementados junto à dieta fracionada, prescritas a uma paciente de cirurgia de hérnia de hiato, e a diminuição do quadro de esofagite. A paciente chegou com quadro de esofagite de refluxo, disfagia, dores na região gástrica e distensão abdominal. Como conduta foi adotada a suplementação com piridoxina $30 \mathrm{mg}$, zinco quelato $20 \mathrm{mg}$ e ácido pantotênico $20 \mathrm{mg}$ e vitamina C revestida $200 \mathrm{mg}$, assim como aumento do fracionamento da dieta, refletindo na redução do volume das refeições para amenizar desconfortos gástricos. O fracionamento adequado da dieta, a redução do volume das refeições e a suplementação atuam de maneira eficaz na melhoria do quadro da DRGE. Cabe destacar que é papel do nutricionista garantir a adesão do paciente ao tratamento para não haver recidivas de sintomas.
\end{abstract}

PALAVRAS-CHAVE: Esofagite. Fracionamento dietético. Suplementação alimentar.

\section{DIETARY CONDUCT IN THE TREATMENT OF GASTROESOPHAGEAL REFLUX DISEASE - CASE REPORT}

ABSTRACT: The gastroesophageal reflux disease is caused by the reduction of the pressure on the lower esophageal sphincter and could be aggravated by inadequate division of the diet and by the production of species that are reactive to oxygen. Medical and surgical treatments are emphasized, but there are also non-pharmacological therapeutic options, such as vitamins and minerals with antioxidant function, acting as adjuvants in this kind of treatment. Therefore, this paper has the purpose of assessing the functions of supplemented vitamin and minerals with a fractionated diet, prescribed to a surgical patient with hiatal hernia, and the reduction of esophagitis. The patient arrived complaining of esophagitis reflux, dysphagia, pain in the gastric region and abdominal distension. Supplementation with 30mg pyridoxine, $20 \mathrm{mg}$ zinc chelate and $20 \mathrm{mg}$ pantothenic acid was prescribed as treatment, as well as $200 \mathrm{mg}$ coated vitamin C tablet, as well as increasing the diet division to reflect the reduction of meal volumes to sooth gastric discomforts. The appropriate fragmentation of the diet, the reduction of meal volumes and the supplementation were effective to improve the GERD condition. It is important to mention that the role of the nutritionist is to ensure the patient adheres to the treatment in order to prevent symptom relapse.

KEYWORDS: Dietary fractioning. Esophagitis. Food supplementation.

\section{Introdução}

O trato gastrintestinal fornece de maneira contínua todos os nutrientes que o organismo necessita para manter suas funções. Isso acontece através da digestão e absorção de nutrientes, todas essas ações são controladas pelo sistema nervoso e pelo sistema hormonal. O que determina o aproveitamento total de um nutriente é o tempo de permanência deste em cada segmento do trato gastrintestinal (GUYTON;
HALL, 2011).

Por meio das ondas peristálticas o esôfago conduz o alimento, que foi deglutido, da faringe até o estômago, causando um relaxamento no estômago e preparando-o para receber o alimento. Na porção inferior do esôfago encontra-se uma área que, diferente das outras porções do esôfago, permanece em contração tônica para evitar o refluxo do conteúdo do estômago para o esôfago, chamada de esfíncter esofágico inferior (GUYTON; HALL, 2011). A redução na pres-

DOI: https://doi.org/10.25110/arqsaude.v20i3.2016.5902

${ }^{1}$ Nutricionista, Mestranda do Programa de Pós-graduação em Ciência Animal com Ênfase em Produtos Bioativos da Universidade Paranaense (UNIPAR), Professora do Curso de Nutrição, Educação Física e Agronomia da Universidade Paranaense (UNIPAR), Sede, Praça Mascarenhas de Moraes, n. 4282, Zona III, CEP. 87.502-210, Umuarama - PR, Brasil - Bolsista do PIT da Universidade Paranaense - UNIPAR. E-mail. tatianesantos@prof.unipar.br - *Autor para correspondência. (44) 998330203

${ }^{2}$ Médica Veterinária, Doutoranda do Programa de Pós-graduação em Ciência Animal com Ênfase em Produtos Bioativos da Universidade Paranaense (UNIPAR). Professora do Curso de Medicina Veterinária da Universidade Paranaense (UNIPAR), Campus II, Rodovia PR 480, Km 14, Parque Bonfim, CEP. 87.502-970, Umuarama - PR, Brasil. E-mail. patriciafranco@prof.unipar.br (44) 991214555

${ }^{3}$ Bióloga, Pós-doutoranda do Programa de Pós-graduação em Ciência Animal com Ênfase em Produtos Bioativos da Universidade Paranaense (UNIPAR), Sede, Praça Mascarenhas de Moraes, n. 4282, Zona III, CEP. 87.502-210, Umuarama - PR, Brasil - Bolsista PNPD/Capes. E-mail. andasoares7@gmail. com (44) 984050149

${ }^{4}$ Acadêmica do curso de Nutrição da Universidade Paranaense (UNIPAR), Sede, Praça Mascarenhas de Moraes, n. 4282, Zona III, CEP. 87.502-210, Umuarama - PR, Brasil. E-mail. maressar8@gmail.com (44) 998776334

${ }^{5}$ Acadêmica do curso de Nutrição da Universidade Paranaense (UNIPAR), Sede, Praça Mascarenhas de Moraes, n. 4282, Zona III, CEP. 87.502-210, Umuarama - PR, Brasil. E-mail. laura.betineli@hotmail.com (44) 999965140

${ }^{6}$ Biólogo, Doutor, Professor do Curso de Medicina Veterinária, Nutrição e Enfermagem e do Programa de Pós-graduação em Ciência Animal com Ênfase em Produtos Bioativos da Universidade Paranaense (UNIPAR), Sede, Praça Mascarenhas de Moraes, n. 4282, Zona III, CEP. 87.502-210, Umuarama - PR, Brasil. E-mail. germano@prof.unipar.br(44) 988038834 
são do esfíncter esofágico inferior deixa a mucosa do esôfago exposta à acidez do estômago podendo levar ao desenvolvimento da doença do refluxo gastroesofágico (DRGE).

Para diagnosticar a DRGE é necessário o conhecimento das manifestações clínicas, as mais comuns são pirose e regurgitação. No entanto, pacientes que apresentam úlcera péptica, gastrite e neoplasias podem apresentar os mesmos sintomas da DRGE, porém a possibilidade do paciente que apresenta essas patologias desenvolver refluxo é acima de $90 \%$. Outras manifestações clínicas, como dor no tórax que não esteja relacionado com problemas coronários, tosse, asma, disfonia e pigarro, estão relacionados também ao refluxo gastroesofágico mesmo que o paciente não apresente os sintomas clássicos (NASI; MORAES-FILHO; CECCONELLO, 2006).

O estômago produz ácido, muco, bicarbonato e anticorpos para proteção contra micro-organismos ingeridos com os alimentos. $\mathrm{O}$ ácido clorídrico é agressor à túnica mucosa e por esse motivo ela é constituída de muco e bicarbonato para sua proteção, como também das células do epitélio (BIGHETTI; ANTÔNIO; CARVALHO, 2002; GUYTON; HALL, 2011).

O tratamento da DRGE consiste em medidas comportamentais e terapêuticas, mesmo não havendo estudos baseados em evidências mantidas por critérios de medicina, a prática clínica tem demonstrado sucesso em sua utilização (NASI; MORAES-FILHO; CECCONELLO, 2006). O uso de vitaminas e minerais com potencial antioxidante também podem ser utilizados como coadjuvantes no tratamento, corroborando com estudos que mostram o envolvimento de espécies reativas ao oxigênio (EROS) na evolução de lesões gástricas (ODABASOGLU et al., 2006; DEVI et al., 2007), sendo que sua produção está associada ao metabolismo celular, mas quando em excesso podem causar lesões oxidativas e morte celular (POTRICH, 2009).

Este relato tem como objetivo avaliar os efeitos da suplementação dietética com vitaminas e minerais e o fracionamento alimentar em uma paciente pós-cirúrgica de hérnia de hiato, e controle dos sintomas da doença do refluxo gastroesofágico.

\section{Relato de Caso}

E. M. B., gênero feminino, 34 anos, atendida no Ambulatório Santa Terezinha, localizado na cidade de Tapejara estado do Paraná, foi atendida após uma cirurgia na junção gastroesofágica, para correção de hérnia de hiato, tratamento de refluxo gastroesofágico. No primeiro atendimento em 30/12/2013, a paciente se apresentou com queixas de dores na região gástrica, distensão abdominal, indigestão e disfagia, dificuldade para engolir, e letargia. Ao diagnóstico nutricional apresentou índice de massa corporal (IMC) 29,42 $\mathrm{kg} / \mathrm{m}^{2}$ que caracteriza pré-obesidade e relação cintura quadril (RCQ) 0,87 que indica risco muito alto de desenvolver doenças associadas à obesidade. Nesta primeira consulta a paciente, foi orientada a aumentar o fracionamento da refeição e diminuir a quantidade de alimento ingerida. Em combinação à prática mencionada, foi realizada a prescrição dietética com a suplementação de piridoxina $30 \mathrm{mg}$, zinco quelato $20 \mathrm{mg}$, ácido pantotênico $20 \mathrm{mg}$ e vitamina C revestida 200 mg (PADOVANI et al., 2006; MAHAN; ESCOTT-STUMP;
RAYMOND, 2013) para que fossem ingeridos concomitante com o almoço por um período inicial de 45 dias .

No dia 28/01/2014, no retorno agendado para receber o plano alimentar, foi relatado melhora nas dores na região gástrica, na digestão e com sensação de redução da distensão abdominal. A perda de massa corporal foi de $1 \mathrm{~kg}$, cabendo destacar que neste período foi realizada apenas a ingestão do suplemento alimentar, e o fracionamento das refeições.

Após longo período sem retorno para acompanhamento do plano alimentar, no dia 23/06/2014, a paciente queixou-se da presença de refluxo gastroesofágico durante a noite, ao ser inquerida sobre a manutenção do plano alimentar e do fracionamento das refeições, relatou a descontinuidade do plano proposto e o aumento do volume de alimentos ingeridos, inclusive no período noturno. Durante os meses em que se manteve afastada do tratamento, a paciente relatou que ingeriu a suplementação durante 45 dias, seguindo a prescrição recebida na consulta do dia 30/12/2013, apresentando melhora dos sintomas neste período. Porém, quando não seguidas as orientações propostas para o fracionamento, temperatura e suplementação dietética, apresentava recidiva dos sintomas da esofagite de refluxo. Seguindo o preconizado anteriormente a paciente foi orientada a retornar ao fracionamento das refeições e a evitar a ingestão de alimentos quentes, principalmente no período noturno.

Em novo retorno no dia 24/11/2014 relatou que não seguiu corretamente as orientações e os sintomas de dores na região gástrica reincidiram e novamente foi prescrita a suplementação com piridoxina $30 \mathrm{mg}$, zinco quelato $20 \mathrm{mg}$, ácido pantotênico $20 \mathrm{mg}$ e vitamina C revestida $200 \mathrm{mg}$ para ingerir com o almoço por 45 dias. No dia 28/11/2014 foi submetida a um exame de vídeo endoscopia que descreveu o esôfago com calibre, distensibilidade e mucosa preservada em todo órgão. Estômago em boas condições para o exame. Lago mucoso claro e em pequena quantidade. Expansibilidade preservada ausência de lesões ulceradas ou tumorais. À retroversão observa-se fundo em disposição de plicatura mucosa de antro com enantema e edema. Piloro centrado e pérvio. O duodeno apresenta forma expansibilidade e mucosa preservada. O diagnóstico foi de fundoplicatura, gastrite endoscópica enantemática leve de antro.

Em 18/12/2014 a paciente, em uso da prescrição dietética, apresentou melhora dos sintomas do refluxo gastroesofágico, contudo, não houve perda de peso que pudesse justificar a melhora dos sintomas, mas sim as alterações dos hábitos alimentares. Nesta consulta foi enfatizada a importância da adesão ao tratamento nutricional, diante das recidivas de sintomas que se apresentaram sempre que a mesma se desviou da conduta dietética planejada.

No dia 16/02/2015 a paciente retornou para o acompanhamento, relatando dificuldade em se adaptar à proposta alimentar devido à rotina de trabalho, neste dia foram feitas alterações no plano alimentar para adaptação aos novos horários, para que se pudessem manter os mesmos princípios de fracionamento e quantidade.

A última consulta registrada foi em 23/03/2015, quando foi relatado pela paciente não estar seguindo o plano alimentar corretamente, exceto quando observava piora dos sintomas do refluxo gastroesofágico. Neste período retornava ao programa alimentar proposto realizando a adequação 
do fracionamento e da quantidade orientada, como também o uso da suplementação prescrita.

\section{Discusão}

O aumento no fracionamento da dieta está diretamente associado a riscos menores de desenvolver obesidade, visto que valores elevados de IMC estão relacionados com um menor fracionamento da alimentação (HOLMBÄCK et al., 2010; BACHMAN et al., 2011).

O Ministério da Saúde preconiza em sua diretriz contida nos "Dez passos para uma alimentação saudável", que ao longo do dia, devem ser realizados três grandes refeições, que devem estar intercaladas com lanches (BRASIL, 2014). Pereira et al. (2014) em estudo realizado com 342 mulheres, revelou que $52 \%$ destas não realizavam o fracionamento adequado da dieta e apresentavam hipertensão arterial, constipação intestinal e baixa ingestão de frutas e água. Ainda segundo os autores, quando não há um fracionamento adequado da alimentação, a quantidade de alimentos que serão ingeridos nas grandes refeições é maior, acarretando aumento na absorção de glicose e lipogênese com consequente aumento da gordura corporal e do peso.

Para que o fracionamento da alimentação seja eficaz, é necessária a adesão e a dedicação por parte de quem o faz, portanto, quando há adesão ao tratamento, cria-se um vínculo entre quem cuida e quem é cuidado, facilitando a conquista do objetivo da intervenção (SILVEIRA; RIBEIRO, 2005).

Para uma orientação individualizada são necessárias várias estratégias e habilidades do nutricionista, tais como: oferecer orientações sobre o tratamento nutricional proposto, sempre esclarecendo possíveis informações distorcidas, com o fornecimento de diretrizes dietéticas e incentivar o paciente para que possa identificar os fatores que impossibilita o alcance dos objetivos proposto.

A conduta dietoterápica é traçada a partir de avaliação que resulta em um diagnóstico específico e individualizado, sendo considerados diversos aspectos funcionais, como os dados clínicos, bioquímicos, antropométricos e alimentares (CFN, 2014). As recidivas são esperadas quando há mudança de padrões de comportamento por um longo período, e a solução é reiniciar o processo de mudança para atingir o objetivo proposto na intervenção (MAHAN; ESCOTTSTUMP; RAYMOND, 2013).

A DRGE afetando aproximadamente $12 \%$ da população brasileira é sem dúvida, um problema de saúde pública (HENRY, 2014).

O tratamento do refluxo gastroesofágico consiste em mudanças comportamentais e medidas farmacológicas que devem ser orientadas e prescritas em conjunto, durante todas as fases da doença, e a adesão ao tratamento é fundamental para o sucesso das medidas propostas. Dentre as mudanças comportamentais encontram-se elevação da cabeceira da cama, diminuição do uso de cigarro, redução do peso quando apresentar sobrepeso, e evitar refeições volumosas (MORAES-FILHO et al., 2002).

A intervenção nutricional baseia-se na redução de alimentos que são de difícil digestibilidade, flatulentos e fermentáveis. $\mathrm{O}$ fracionamento da alimentação deve ser aumentado a fim de evitar desconforto e o aumento da pressão ab- dominal, já o volume das refeições deve ser diminuído para evitar distensão intra-abdominal e a estimulação do ácido clorídrico. A temperatura não dever ser elevada, visto que alimentos quentes aumentam a congestão da mucosa gástrica, e a secreção ácida, assim como no tempo de esvaziamento gástrico, fazendo com que o alimento fique por mais tempo no estômago (REIS, 2003).

O grande desafio do paciente com a DRGE é a manutenção do tratamento e do quadro assintomático (HENRY, 2014), geralmente os pacientes são mantidos em intervenção farmacológica e acabam abandonando o tratamento com a diminuição ou o desaparecimento dos sintomas.

Além dos procedimentos convencionais farmacológicos, outros tratamentos como a suplementação dietética destaca-se como integrante importante da conduta não farmacológico, sendo eles capazes de promover melhora dos sintomas com a mesma eficácia de um fármaco, porém sem causar os efeitos colaterais. A ação antioxidante de alguns suplementos e vitaminas refletem na melhora do organismo de forma sistêmica e não unicamente em uma doença, tornando seu uso uma opção a ser adotada.

O zinco representa um mineral com importante ação na função imune, pois participa ativamente da formação da enzima superóxido dismutase que é um antioxidante. Diversos estudos tem destacado a importância do zinco na alimentação, principalmente por sua participação no mecanismo de defesa antioxidante, sendo que a suplementação com esse mineral tem mostrado resultados promissores no tratamento da diarreia e infecções oportunistas em pacientes aidéticos (FRAKER et al., 2000; MAFRA; COZZOLINO, 2004).

A redução nos sintomas da esofagite após a suplementação com zinco, como relatada neste estudo, sugere que este mineral está impactando de forma positiva no sistema digestório, pela melhora na resposta inflamatória ocasionada pelo refluxo gastroesofágico.

$\mathrm{O}$ ácido ascórbico ou vitamina $\mathrm{C}$ é um sólido cristalino branco muito solúvel em água que participa da absorção do ferro, síntese de colágeno, assim como na integridade do tecido conjuntivo participando de processos de cicatrização e também é considerado um composto antioxidante que atua no sistema imunológico reduzindo as EROS em leucócitos ativados no pulmão e na mucosa gástrica. Os neutrófilos são células fagocíticas do sistema imune que quando ativadas, provocam uma liberação excessiva de EROS que resulta em danos no tecido, potencialização no processo inflamatório de algumas patologias, portanto, o ácido ascórbico reduz a suscetibilidade às infecções e inflamações (ARANHA et al., 2000; HALLIWELL, 2001; REIS, 2003; CERQUEIRA; MEDEIROS; AUGUSTO, 2007; FUZISSAKI, 2009), contribuindo para a redução do quadro de esofagite descrita pela paciente.

As vitaminas do complexo $\mathrm{B}$ como a vitamina $\mathrm{B}_{6}$ em suas formas ativas são coenzimas na transaminação e na desaminação, e também está envolvida no metabolismo de carboidratos e lipídeos, assim como, no processo antioxidante, neutralizando radicais livres com a mesma eficácia dos tocoferóis e carotenóides. A piridoxina faz parte de uma enzima antioxidante muito importante, a glutationa peroxidase. A relevância dessa vitamina no sistema imune está relacionada ao seu papel na biossíntese de proteínas, visto que os anticorpos e citocinas, atuantes no processo inflamatório, 
são formados à base de aminoácidos (PASCHOAL; NAVES; FONSECA, 2007; COZZOLINO; COMINETTI, 2013).

Outra vitamina do complexo B, o ácido pantotênico ou vitamina $\mathrm{B}_{5}$, apresenta-se na forma ativa como coenzima A, desempenhando importante papel na formação da molécula de Acetil CoA, principal componente do ciclo do ácido cítrico. A vitamina $B_{5}$ também é necessária para síntese dos fosfolipídios de membrana, aminoácidos como a leucina arginina e metionina (MAHAN; ESCOTT-STUMP; RAYMOND, 2013; BIEZEK; ALVES; GUERRA, 2015).

Todas as medidas terapêuticas buscam melhorar a função motora do esôfago, aumentar a pressão do esfíncter esofágico inferior, assim como reduzir o prejuízo causado pela acidez gástrica, neutralizando-a ou mesmo suprimindo-a, contudo, como já mencionado, o maior empecilho no tratamento é manter os pacientes assintomáticos, visto que mesmo após o tratamento cirúrgico feito através de laparoscopia, ainda há queixas reincidentes de disfagia, dificuldade para eructar, flatulência e diarreia (KLAUS et al., 2003; PEREIRA, et al., 2007; ANDRADE et al., 2012).

\section{Conclusão}

O uso de suplementos vitamínicos e minerais para o tratamento da DRGE demonstrou ação eficaz na diminuição dos sintomas da doença. Para que o paciente permaneça assintomático, é necessário associar à suplementação, um fracionamento adequado da dieta, assim como a redução do volume ingerido em cada refeição. O nutricionista deve fornecer apoio para a adesão do paciente ao tratamento, visto que a conduta proposta é fundamental para que não haja interrupções e recidivas dos sintomas.

\section{Referências}

ANDRADE, F. J. C. et al. Qualidade de vida do paciente submetido à cirurgia videolaparoscópica para tratamento para doença do refluxo gastroesofágico. ABCD. Arquivos Brasileiros de Cirurgia Digestiva (São Paulo), v. 25, n. 3, p. 154-160, 2012.

ARANHA, F. Q. et al. O papel da vitamina C sobre as alterações orgânicas no idoso. Revista de Nutrição, v. 13, n. 2, p. 89-97, 2000.

BACHMAN, J. L. et al. Eating frequency is higher in weight loss maintainers and normal-weight individuals than in overweight individuals. Journal of the American Dietetic Association, v. 111, n. 11, p. 1730-1734, 2011.

BIESEK, S.; ALVES, A. L.; GUERRA, I. Estratégias de suplementação no esporte. 3. ed. Barueri: Manole, 2015. $460 \mathrm{p}$.

BIGHETTI, A. E.; ANTÔNIO, M. A.; CARVALHO, J.E. Regulação e modulação da secreção gástrica. Revista de Ciências Médicas, v. 11, n. 1, p. 55-60, 2002.

BRASIL. Ministério da Saúde. Guia alimentar para a população brasileira: promovendo a alimentação saudável. Brasília: Ministério da Saúde, 2014.
CERQUEIRA, F. M.; MEDEIROS M. H. G.; AUGUSTO, O. Antioxidantes dietéticos: controvérsias e perspectivas. Química Nova, v. 30, n. 2, p. 441, 2007.

\section{CONSELHO FEDERAL DE NUTRIÇÃO (CFN).}

Resolução CFN n. 514, de 14 de maio de 2014. Diário Oficial da União, Brasília, 19 maio 2014. Seção 1, p.131.

\section{COZZOLINO, S. M. F.; COMINETTI, C. Bases}

bioquímicas e fisiológicas da nutrição: nas diferentes fases da vida, na saúde e na doença. Barueri: Manole, 2013. $1288 \mathrm{p}$.

DEVI, R. S. et al. Gastroprotective effect of Terminalia arjuna bark on diclofenac sodium induced gastric ulcer. Chemico-biological interactions, v. 167, n. 1, p. 71-83, 2007.

FRAKER, P. J. et al. The dynamic link between the integrity of the immune system and zinc status. The Journal of Nutrition, v. 130, n. 5, p. 1399S-1406S, 2000.

FUZISSAKI, C. N. Estudo da modulação de funções efetoras de neutrófilos humanos por derivados cumarínicos: avaliação do efeito biológico sobre a produção de espécies reativas de oxigênio e a desgranulação. 2009. 42 f. Dissertação (Mestrado) Faculdade de Ciências Farmacêuticas de Ribeirão Preto, Universidade de São Paulo, Ribeirão Preto, 2009.

GUYTON, A.C.; HALL, J. E. Tratado de fisiologia médica. Tradução Alcides Martins Junior et al. 12 ed. Rio de Janeiro: Elsevier; 2011. 1151 p.

HALLIWELL, B. Vitamin C and genomic stability. Mutation Research/ Fundamental and Molecular Mechanisms of Mutagenesis, v. 475, n. 1, p. 29-35, 2001.

HENRY, M. A. C. A. Diagnóstico e tratamento da doença do refluxo gastroesofágico. Arquivos Brasileiros de Cirurgia Digestiva, v. 27, n. 3, p. 210-215, 2014.

HOLMBÄCK, I. et al. A high eating frequency is associated with an overall healthy lifestyle in middle-aged men and women and reduced likelihood of general and central obesity in men. British Journal of Nutrition, v. 104, n. 07, p. 1065-1073, 2010.

KLAUS, A. et al. Bowel dysfunction after laparoscopic antireflux surgery: incidence, severity, and clinical course. The American Journal of Medicine, v. 114, n. 1, p. 6-9, 2003.

MAFRA, D.; COZZOLINO, S. M. F. The importance of zinc in human nutrition. Revista de Nutrição, v. 17, n. 1, p. 79-87, 2004.

MAHAN, L. K.; ESCOTT-STUMP, S. RAYMOND, J. L. Krause: alimentos, nutrição e dietoterapia. Tradução Claudia Coana et al. 12. ed. Rio de Janeiro: Elsevier, 2013. $1227 \mathrm{p}$. 
MORAES-FILHO, J. P. P. et al. Brazilian Consensus on Gastroesophageal Reflux Disease: proposals for assessment, classification, and management. The American Journal of Gastroenterology, v. 97, n. 2, p. 241-248, 2002.

NASI, A.; MORAES-FILHO, J. P. P.; CECCONELLO, I. Doença do refluxo gastresofágico: revisão ampliada. Arquivos de Gastroenterologia, v. 43, n. 4, p. 334-341, 2006.

ODABASOGLU, F. et al. Gastroprotective and antioxidant effects of using acid on indomethacin-induced gastric ulcer in rats. Journal of Ethnopharmacology, v. 103, n. 1, p. 59-65, 2006.

PADOVANI, R. M. et al. Dietary reference intakes: aplicabilidade das tabelas em estudos nutricionais. Revista de Nutrição, v. 19, n. 6, p. 741-760, 2006.

PASCHOAL, V.; NAVES, A.; FONSECA, A. B. L. Nutrição clínica funcional: dos princípios à prática clínica. 2. ed. São Paulo: Valéria Paschoal Editora, 2007.328 p.

PEREIRA, G. I. N. et al. Tradução e validação para a língua portuguesa (Brasil) de instrumentos específicos para avaliação de qualidade de vida na doença do refluxo gastroesofágico. Arquivos de Gastroenterologia, v. 44, n. 2, p. 168-177, 2007.

PEREIRA, L. M. et al. Fracionamento da dieta e o perfil nutricional e de saúde de mulheres. Revista de Nutrição, v. 27 , n. 1, p. 15-2, 2014.

\section{POTRICH, F. B. Atividade gastroprotetora do} extrato bruto hidroalcoólico da Achilea millefolium L.: envolvimento do sistema antioxidante. 2009. $67 \mathrm{f}$. Dissertação (Mestrado em Farmacologia) - Setor de Ciências Biológicas, Universidade Federal do Paraná, Curitiba, 2009.

REIS, N. T. Nutrição clínica: sistema digestório. Rio de Janeiro: Rubio, 2003. 294 p.

SILVEIRA, L. M. C., RIBEIRO, V. M. B. Grupo de adesão ao tratamento: espaço de "ensinagem" para profissionais de saúde e pacientes. Interface-Comunicação, saúde, educação, v. 9, n. 16, p. 91-104, 2005. 